\title{
La Eutrofización acuática y terrestre como categorías de impacto regional
}

\section{Aquatic and terrestrial eutrophication as regional impact categories}

Presentación: 6-7/10/2020

\author{
Doctorando: \\ Eliana Conci \\ Grupo CLIOPE "Energía, Ambiente y Desarrollo Sustentable", Facultad Regional Mendoza, Universidad Tecnológica Nacional - \\ Argentina \\ econci@mendoza-conicet.gob.ar
}

\section{Directora:}

\section{Bárbara María Civit}

\section{Co-directores:}

\section{Analía Rosa Becker \\ Alejandro Pablo Arena}

\begin{abstract}
Resumen
La creciente conciencia con respecto a la importancia de la protección ambiental y los posibles impactos asociados con los productos han aumentado el interés por el desarrollo de métodos para comprender y tratar los mismos, tal como, el Análisis de Ciclo de Vida (ACV). Uno de los riesgos que deriva de las diversas actividades económicas agricultura, ganadería, industria, comercio, urbanización, otras- es la eutrofización de cuerpos de agua y del suelo, y estas categorías son consideradas en todos los modelos de Evaluación del Impacto del Ciclo de Vida (EICV). Sin embargo, la Eutrofización acuática (agua dulce y marina) y terrestre aún no está explorada en el ámbito del ACV a nivel país. El plan de Tesis Doctoral tiene como objetivo desarrollar indicadores regionales de Eutrofización, profundizando en el estudio de la degradación del suelo y agua, bajo la metodología del ACV. Hasta el momento, se ha efectuado una revisión exhaustiva de la bibliografía y de antecedentes específicos en el plano nacional e internacional sobre estas categorías de impacto regional. Se observa que hay escasos artículos de investigación que analizan de manera integrada los ecosistemas acuáticos y terrestres, y que en Argentina se requieren estudios sobre Eutrofización en regiones con significancia ambiental, dada la variabilidad bioclimática y el sistema productivo, con factores de caracterización (CFs) dependientes del sitio. Se prevé efectuar un análisis sistemático de los estudios resultantes de la revisión bibliográfica que permitan identificar las necesidades de investigación para la región y aporten una base sólida para la continuidad de los objetivos planteados. La obtención de un conjunto de indicadores contribuiría a la sustentabilidad ambiental de Argentina, brindando herramientas para minimizar los impactos derivados de la producción y el consumo de productos y servicios.
\end{abstract}

Palabras clave: nutrientes, eutrofización, impactos ambientales, regionalización, Análisis de Ciclo de Vida.

\begin{abstract}
The growing awareness of the importance of environmental protection and the potential impacts associated with products have increased interest in developing methods to understand and address them, such as, Life Cycle Assessment (LCA). One of the risks arising from various economic activities - agriculture, livestock, industry, commerce, urbanization, others - is the eutrophication of water bodies and soil, and these categories are considered in all Life Cycle Impact Assessment (LCIA) models. However, aquatic (freshwater and marine) and terrestrial eutrophication is still unexplored in the field of LCA at the country level. The Doctoral Thesis plan aims to develop regional indicators of Eutrophication, deepening the study of soil and water degradation, under the methodology of LCA. So far, a comprehensive review of the literature and specific background at the national and international level on these regional impact categories has been conducted. It is observed that there are few research articles that analyze
\end{abstract}


in an integrated way the aquatic and terrestrial ecosystems, and that in Argentina studies on Eutrophication in regions with environmental significance are required, given the bioclimatic variability and the productive system, with sitedependent characterization factors (CFs). A systematic analysis of the studies resulting from the literature review is planned to identify research needs for the region and provide a solid basis for the continuity of the objectives set. Obtaining a set of indicators would contribute to Argentina's environmental sustainability, providing tools to minimize the impacts derived from the production and consumption of products and services.

Keywords: nutrient, eutrophication, environmental impacts, regionalization, Life Cycle Assessment.

\section{Introducción}

Un sistema de producción obedece a múltiples interacciones entre el aprovechamiento de los recursos naturales y la tecnología aplicada, por lo que es necesario comprender el grado de fragilidad de los sistemas para evitar llegar a extremos de deterioro (Fernández \& Urricariet, 2014). En la naturaleza los procesos de degradación física, química y biológica actúan en forma combinada y aumentando su efecto negativo sobre los recursos naturales (Pla Sentís, 1990). La consolidación de un mercado global ha establecido relaciones entre las economías de los países a través de cadenas de suministro que elaboran productos y servicios con repercusiones en todos los ámbitos de la sustentabilidad: económico, ambiental y social. En este contexto, las herramientas que actúan en el marco del pensamiento de ciclo de vida han adquirido una gran relevancia (Perez, 2016), puesto que permiten determinar el perfil ambiental del sistema evaluado y producir información sobre la cuantificación y origen de los recursos materiales y energéticos (Hilbert \& Schein, 2018).

La metodología del ACV cobró relevancia en la década del noventa y se ha ido desarrollando y mejorando mediante el aporte de diferentes investigaciones. A fin de reforzar esta herramienta, Potting \& Hauschild (2006) reconocieron que para muchas categorías (intervenciones ambientales), el impacto de un determinado elemento depende de dónde se produce ese flujo y por lo tanto tiene CFs (o indicadores) dependientes del sitio. Los factores regionales se calculan para categorías de impacto producidas por emisiones de sustancias que tienen tiempos de vida relativamente cortos y que son transportadas cientos o miles de kilómetros. Entre ellas se encuentran las categorías que miden la acidificación, la Eutrofización acuática y terrestre, la formación de oxidantes fotoquímicos o la toxicidad (Antón, 2004; Udo de Haes et al., 2002). Civit (2009) expresa que según sea la escala geográfica en la que actúan o tienen influencia los efectos del impacto considerado, se puede dividir a las categorías de impacto en globales, regionales o locales e indica la importancia de la incorporación de las características locales y regionales para evaluar indicadores. En el último decenio la regionalización de los métodos de evaluación de impacto ha incluido categorías como la Eutrofización, considerada en todos los modelos de EICV, una de las etapas de un ACV.

En Latinoamérica, y específicamente en Argentina, existen algunos antecedentes de desarrollo de indicadores de impacto para categorías con carácter regional: Uso del agua en la cadena vitivinícola (Civit et al., 2018), Acidificación terrestre (Civit et al., 2014), Desertificación (Civit et al., 2013; Núñez et al., 2010), Eutrofización terrestre y Uso del Suelo (Civit, 2009). No obstante, la Eutrofización acuática (agua dulce y marina) y terrestre aún no está explorada en el ámbito del ACV a nivel país, si en algunos sectores específicos, como Mendoza. Dentro de este contexto, el plan de Tesis Doctoral tiene como objetivo desarrollar indicadores regionales de Eutrofización, profundizando en el estudio de la degradación del suelo y agua. La investigación se enmarca dentro del conocimiento en el campo de la sustentabilidad ambiental regional, brindando herramientas para minimizar el riesgo ambiental, profundizando en el estudio sobre los efectos potenciales que podrían producirse por la emisión de sustancias eutrofizantes al aire, suelo y agua a lo largo del ciclo de vida de productos, procesos o actividades. A fin de llevar adelante el objetivo principal, se plantean los siguientes objetivos específicos:

a. Seleccionar un modelo de impacto de punto intermedio para ecosistemas terrestres y acuáticos regionales;

b. Regionalizar los mecanismos de impacto de Eutrofización acuática y terrestre para los principales ecosistemas receptores de Argentina;

c. Determinar CFs para Eutrofización acuática y terrestre regional;

d. Proponer un conjunto de indicadores regionales para Eutrofización con aplicabilidad en la evaluación ambiental a lo largo del ciclo de vida de productos que resulten de distintas actividades económicas;

e. Validar los indicadores obtenidos para la Eutrofización acuática y terrestre regional en un ACV concreto.

La metodología de desarrollo a seguir se enmarca dentro de la tercera etapa de un ACV, la EICV, según las normas ISO 14040 (ISO, 2006a) y 14044 (ISO, 2006b). 


\section{Desarrollo}

La creciente conciencia con respecto a la importancia de la protección ambiental y los posibles impactos asociados con los productos han aumentado el interés por el desarrollo de métodos para comprender y tratar esos impactos. Una de las técnicas desarrolladas en este sentido es el ACV (ISO, 2006a), que nació en la década del sesenta cuando fue evidente que el único modo eficaz de analizar los sistemas industriales era el de examinar todos los procesos seguidos por la materia prima, desde su extracción, siguiendo con los procesos de transformación y terminando con el retorno a la ecosfera en forma de residuos (Arena, 1999). No obstante, es en la década de 1990 cuando se produce la consolidación de esta metodología (Schmid, 2008).

Los factores regionales no están desarrollados para todas las partes del mundo (normalmente sólo para Europa y América del Norte) (Potting et al., 2001), lo que es un problema en un mundo globalizado donde los procesos que componen un sistema pueden tener lugar en cualquier parte del planeta (Schmid, 2008). Ante esto, existen iniciativas de desarrollo del ACV en Argentina y son promovidas por los diversos encuentros científicos que se llevan a cabo tanto en el país como en Latinoamérica. Si bien el desarrollo de estos temas tiene ya varios años, persiste aún la necesidad a nivel nacional de validar y adecuar en forma consensuada los desarrollos metodológicos internacionales con el propósito de proporcionar información ambiental consistente de productos nacionales (Hilbert \& Schein, 2018). Es por ello que a la hora de evaluar los impactos es necesario contar con factores que caractericen el efecto potencial que la intervención ambiental tiene en un sitio y no en otro. Asimismo, para garantizar que los resultados de un ACV sean coherentes, los métodos y sus modelos deben actualizarse continuamente y la tendencia es regionalizar CFs (Morelli et al., 2018; Civit, 2009; Potting \& Hauschild, 2006).

Actualmente, la Iniciativa del Ciclo de Vida auspiciada por ONU-Medio Ambiente inicio un proceso mundial en 2013 a través de grupos de trabajo internacionales para llegar a un consenso sobre indicadores ambientales y CFs recomendados para la EICV. Entre 2016 y 2018 se repitió el mismo proceso, pero esta vez incluyó otros temas como la Eutrofización de agua dulce y marina. La Eutrofización era considerada como una única categoría de impacto. Fue recién en 1992 que se explicitó la diferencia entre Eutrofización terrestre y acuática, debido a que los ecosistemas terrestres se ven afectados por el aporte de las emisiones de compuestos de $\mathrm{N}$ (óxidos de $\mathrm{N}$ y amoníaco) emitidos en el aire durante todo el ciclo de vida de productos, procesos o actividades, por deposición atmosférica o nitrificación (Civit, 2009).

Por los motivos expuestos, como resultado de la Tesis Doctoral se espera obtener un conjunto de CFs para determinar el impacto por Eutrofización acuática y terrestre en Argentina. De esta manera, se intenta contribuir a la sustentabilidad ambiental regional, otorgando herramientas para minimizar el riesgo ambiental de la producción y el consumo de productos y servicios. En estos primeros meses, se ha avanzado en la búsqueda de información, recopilando datos necesarios y detectando faltantes, con la finalidad de conocer el estado del arte del tema en cuestión, los posibles nichos de investigación y resultados potenciales. La revisión bibliográfica se efectuó a través de la búsqueda de diferentes trabajos académicos, mediante los siguientes buscadores: Google Scholar, Science Direct, Springer Link, Scielo, REDIB, OAJI, DOAJ, JSTOR, Latindex, Publindex, Dialnet y Redalyc. Así mismo, se revisaron actas de eventos científicos, Encuentros tales como ENARCIV, ASADES, ICES, ENIDI y páginas de la FAO, SETAC, INTA, Life Cycle Initiative, entre otras vinculadas a la temática. Previamente, se determinaron palabras clave como estrategia de búsqueda, usando su traducción al inglés en aquellos buscadores que lo requerían. Las mismas se combinaron en los campos "título", "resumen" y "palabras clave" considerando trabajos publicados entre 1990 (consolidación del ACV) y 2020. Cabe destacar que en el campo "sin las palabras" se expusieron "metales pesados, construcción y energía eléctrica", ya que los buscadores arrojaban resultados que no se relacionaban directamente con el objetivo de la investigación. Las palabras seleccionadas fueron:

1. Eutrofización y Ciclo de Vida (o Análisis de Ciclo de Vida)

2. Eutrofización y Evaluación del Ciclo de Vida (o Evaluación del Impacto del Ciclo de Vida)

3. Eutrofización e indicadores

4. Eutrofización acuática y Ciclo de Vida

5. Eutrofización marina y Ciclo de Vida

6. Eutrofización terrestre y Ciclo de Vida

7. Eutrofización e indicadores de impacto regional

8. Regionalización de impactos y Ciclo de Vida

Para la selección de la bibliografía adecuada, primeramente se revisaron los títulos, resúmenes y palabras clave, de modo de identificar el tipo de información que contenía cada una. A partir de la búsqueda inicial, finalmente se seleccionaron 102 estudios desarrollados en el ámbito del ACV, entre los que se encuentran artículos de investigación, 
de revisión, discusiones, revisiones críticas, informes de reuniones, tesis doctorales, cartas al editor, guías globales, libros y capítulos, apoyo científico-técnico, resúmenes en eventos científicos e informe de metodología.

Hasta el momento, se observa que hay escasos trabajos de investigación que analizan las categorías Eutrofización acuática y terrestre en un mismo estudio. Si se encuentra el análisis de la Eutrofización acuática, ya sea de agua dulce o marina, por un lado, y la terrestre por el otro (Tabla 1 y Figura 1).

Cantidad de trabajos publicados en función del tema

Serie 1990-2020

\begin{tabular}{cccc}
\hline & Temas & Abreviaturas & \multicolumn{2}{c}{ Cantidad de trabajos } \\
\hline & Análisis de Ciclo de Vida & ACV & 23 \\
\hline & Evaluación del Impacto del Ciclo de Vida & EICV & 13 \\
\hline \multirow{2}{*}{$\begin{array}{c}\text { En el contexto } \\
\text { del ACV }\end{array}$} & Eutrofización & E & 6 \\
& Eutrofización acuática de agua dulce & EA & 13 \\
& Eutrofización marina & EM & 8 \\
& Eutrofización terrestre & ET & 6 \\
& Mefización acuática y Eutrofización terrestre & EA+ET & 1 \\
\hline & Métodos & M & 25 \\
\hline
\end{tabular}

Tabla 1: Cantidad de trabajos publicados en función del tema. Serie 1990-2020.

Los trabajos seleccionados referidos a "ACV" son aquellos que otorgan un marco de análisis en función del estudio de la Eutrofización y que brindan antecedentes sólidos para el desarrollo de la Tesis y la puesta en contexto como estado del arte. Es por ello que se excluyeron aquellos que no guardaban una relación directa con la categoría de impacto en estudio.

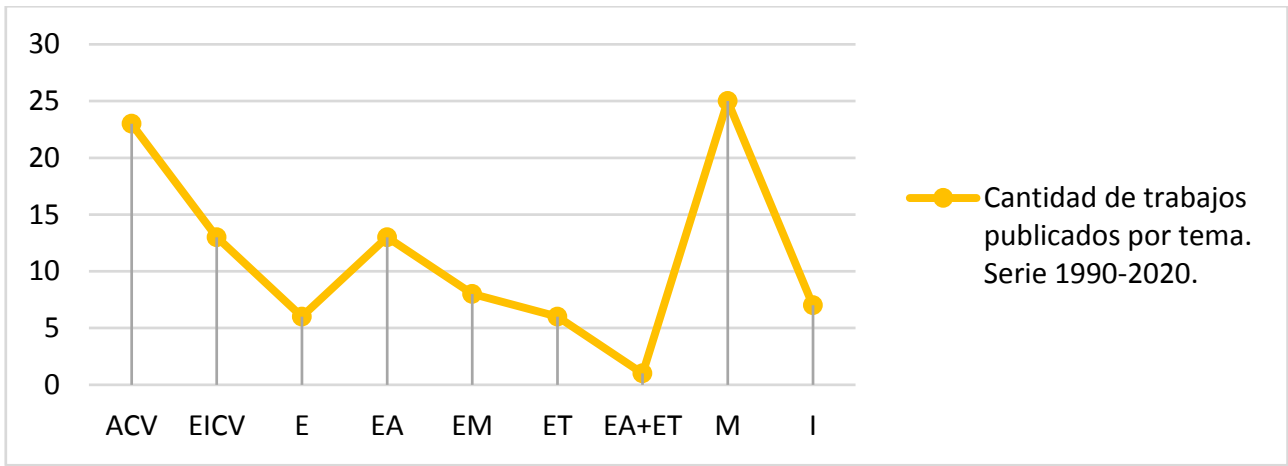

Figura 1: Cantidad de trabajos publicados por tema. Serie 1990-2020.

A medida que la caracterización de los impactos ha ido madurando, el estudio de los factores específicos de caracterización se ha reducido a escala regional (especialmente en los países que tienen una gran variabilidad geográfica, climática y económica) siendo un foco de interés en las diferentes categorías de impacto de la EICV (Fréchette-Marleau et al., 2008; Toffoletto et al., 2007; Hauschild \& Potting, 2005). Por ello, se han comenzado a difundir metodologías de EICV más sofisticadas que tienen en cuenta no sólo la cantidad de emisiones producidas, sino también el lugar donde son liberadas, su destino y el daño que producen, sea sobre el ecosistema natural, artificial o sobre la salud humana. En estas metodologías, los factores de equivalencia utilizados reflejan una dependencia del sitio y serán en general diferentes de un lugar a otro (Civit et al., 2006). El trabajo de Civit (2009) constituye un antecedente primordial en el estudio de Eutrofización terrestre y pone en evidencia que se requieren estudios sobre esta categoría de impacto en Argentina en regiones con significancia ambiental, considerando las características de los ecosistemas receptores y la inclusión del análisis de los mecanismos de impacto en ecosistemas acuáticos. Esto se justifica en que varios autores han estudiado la Eutrofización acuática en la región, pero fuera del contexto del ACV (por ejemplo, Mengo et al., 2019; Espósito et al., 2016; Ledesma et al., 2013) y Quirós (2000) indica que el país presenta graves problemas en algunos de sus lagos y embalses. Esta necesidad se incrementa al considerar que de los 102 trabajos seleccionados, solo 4 se desarrollaron en Argentina (particularmente en la provincia de Mendoza), dentro de los cuales, 3 se encuentran enfocados a la Eutrofización terrestre. 
Las contribuciones humanas al aumento del ciclo del nitrógeno $(\mathrm{N})$ y el fósforo $(\mathrm{P})$ a través de la biosfera amenazan la salud de los ecosistemas de agua dulce y marinos y las funciones económicas y de apoyo a la vida que éstos desempeñan (Rockström et al., 2009). Al comparar los cuerpos de agua dulce o las zonas terrestres, puede haber diferencias debidas a la variación del clima, la composición de las especies, la geología subyacente o las tensiones ambientales previas, entre otras. Muchos de estos factores controlan los niveles existentes de nutrientes; esto puede afectar al que es limitante (Henderson, 2015). Las escalas de tiempo en las que las emisiones eutrofizantes alcanzan y causan impacto en un lugar de recepción varían según el compuesto y el compartimento de emisión (Galloway, 2003). Dado que Argentina presenta una extensa variabilidad bioclimática y que es un país productor de materias primas y productos intermedios que se incorporan a diversos sistemas productivos en distintas partes del mundo, a la hora de evaluar los impactos es necesario contar con factores que caractericen el efecto potencial que la intervención ambiental tiene en un sitio y no en otro. Por ello, debido a la alta variabilidad de los impactos de la Eutrofización (Helmes et al., 2012), los enfoques que proporcionan la diferenciación espacial son preferibles (Payen et al., 2019).

\section{Conclusiones}

La elección del desarrollo de indicadores regionales dentro de la etapa de EICV es visiblemente justificada para llevar adelante una investigación, donde la Eutrofización acuática y terrestre aún no está explorada en el ámbito del ACV a nivel regional. Esta contribución generaría una base de datos técnica y metodológica propia, para la cuantificación de impactos ambientales derivados de diversas actividades económicas, posicionándose como un avance en el estudio de riesgos ambientales de la región.

Considerando la variabilidad bioclimática de Argentina, la contribución de investigadores y profesionales abocados al estudio y aplicación del ACV ponen de manifiesto la necesidad de regionalizar CFs. Por ello, se requieren estudios científicos basados en las características ambientales locales con significancia ambiental, de modo que la presencia de valores regionales evite el uso de CFs desarrollados para otros sitios, generando resultados no representativos para categorías de impacto dependientes del sitio como lo es la Eutrofización. La obtención de un conjunto de indicadores contribuiría a la sustentabilidad ambiental de Argentina, brindando herramientas para minimizar los impactos derivados de la producción y el consumo de productos y servicios.

Para dar continuidad a la investigación, se prevé realizar un análisis sistemático de los estudios resultantes de la revisión bibliográfica, identificando los métodos de EICV utilizados, tipo de diferenciación y agregación, los CFs a nivel de punto medio y final, a fin de efectuar una publicación científica. Estos resultados expresaran el estado del arte de las categorías de impacto ambiental Eutrofización acuática y terrestre, permitirán identificar las necesidades de investigación para la región y aportarán una base sólida para dar seguimiento a los objetivos específicos del plan de Tesis Doctoral.

\section{Referencias}

Antón, M. A. (2004). Utilización del Análisis de Ciclo de Vida en la Evaluación del Impacto Ambiental del Cultivo bajo Invernadero Mediterráneo. Universidad Politécnica de Cataluña. ISBN: 8468867322.

Arena, A. P. (1999). Un instrumento para el análisis y evaluación ambiental de productos y tecnologías. El análisis de ciclo de vida.-Consideraciones metodológicas, usos y limitaciones. Revista de la Asociación Argentina de Energías Renovables y Ambiente, 3(2). ISSN: 0329-5184.

Civit, B. M. (2009). Sostenibilidad ambiental. Desarrollo de indicadores para su aplicación en estudios de análisis de ciclo de vida en la región árida del centro-oeste argentino. Universidad Nacional de Cuyo.

Civit, B. M., Arena, A. P. \& Allende, D. (2014). Determination of regional acidification factors for Argentina. The International Journal of Life Cycle Assessment, 19(9), 1632-1642. https://doi.org/10.1007/s11367-012-0427-6

Civit, B. M., Arena, A. P., Núñez, M., Muñoz, P., Antón, A. \& Rieradevall, J. (2013). Assessing potential desertification environmental impact in life cycle assessment. Part 2: agricultural case study in Spain and Argentina. The International Journal of Life Cycle Assessment, 18(7), 1302-1315. https://doi.org/10.1007/s11367-013-0582-4

Civit, B., Arena, A. P., \& Puliafito, S. E. (2006). Estudio de deposiciones de nitrógeno en suelos para la evaluación de la eutrofización terrestre en la región centro oeste árida argentina. Avances en Energías Renovables y Medio Ambiente, 10. ISSN: 0329-5184.

Civit, B. M., Piastrellini, R., Curadelli, S. \& Arena, A. P. (2018). The water consumed in the production of grapes for vinification (Vitis vinifera). Mapping the blue and green water footprint. Ecological Indicators, 85, $236-243$. https://doi.org/10.1016/j.ecolind.2017.10.037 
Espósito, M. E., Blanco, M. D. C., Sequeira, M. E., Paoloni, J. D., Fernández, S. N., Amiotti, N. M., \& Díaz, S. L. (2016). Contaminación natural (As, F) y eutrofización (N, P) en la cuenca del arroyo El Divisorio, Argentina. Phyton - International Journal of Experimental Botany, 85, 51-62. ISSN: 0031-9457.

Fernández, P. L., \& Urricariet, S. (2014). Deterioro de los recursos naturales. Degradación y calidad de suelos. In P. B. Lombardo, P. L. Fernández \& S. Urricariet. (Eds.), Agroecosistemas. Caracterización, implicancias ambientales y socioeconómicas (1era ed.). Editorial FAUBA. ISBN: 978-987-3738-01-2.

Fréchette-Marleau, S., Bécaert, V., Margni, M., Samson, R., \& Deschênes, L. (2008). Evaluating the variability of aquatic acidification and photochemical ozone formation characterization factors for Canadian emissions. The International Journal of Life Cycle Assessment, 13(7), 593. https://doi.org/10.1007/s11367-008-0027-7

Galloway, J. N. (2003). The global nitrogen cycle. TrGeo, 8, 682. DOI: 10.1016 / B0-08-043751-6 / 08160-3

Hauschild, M., \& Potting, J. (2005). Spatial differentiation in Life Cycle impact assessment-The EDIP2003 methodology. Danish Ministry of the Environment, 80, 1-195.

Helmes, R. J. K., Huijbregts, M. A. J., Henderson, A. D., \& Jolliet O. (2012). Spatially explicit fate factors of phosphorous emissions to freshwater at the global scale. The International Journal of Life Cycle Assessment, 17: 646-654. https://doi.org/10.1007/s11367-012-0382-2

Henderson, A. D. (2015). Eutrophication. In M. Z. Hauschild \& M. A. J. Huijbregts (Eds.), Life Cycle Impact Assessment (pp. 177-196). Springer. DOI: 10.1007/978-94-017-9744-3

Hilbert, J. A., \& Schein, L. (2018). Las huellas ambientales de la generación de valor (1era ed.). Ediciones INTA. http://www.bioeconomia.mincyt.gob.ar/wp-content/uploads/2018/10/ENARCIV-FINAL.pdf

ISO. (2006a). Gestión ambiental - Evaluación del ciclo de vida - Principios y marco. 14040, Organización Internacional de Normalización.

ISO. (2006b). Gestión ambiental - Evaluación del ciclo de vida - Requisitos y directrices. 14044, Organización Internacional de Normalización.

Ledesma, C., Bonansea, M., Rodriguez, C. M., \& Delgado, A. R. S. (2013, jul-set). Determinación de indicadores de eutrofización en el embalse Río Tercero, Córdoba (Argentina). Revista Ciência Agronômica, 44(3), 419-425. ISSN: 1806-6690.

Mengo, L., Lami, A., Guerra, L., Masuzzi, S., Piovano, E. L., \& Halac, S. R. (2019). Paleolimnología del embalse San Roque. Relación entre la eutrofización y la variabilidad hidroclimática. V Reunión Argentina de Geoquímica de la Superficie (RAGSU), 194-197. ISBN: 978-987-96296-7-3.

Morelli, B., Hawkins, T. R., Niblick, B., Henderson, A. D., Golden, H. E., Compton, J. E., Cooter, E. J. \& Bare, J. C. (2018). Critical Review of Eutrophication Models for Life Cycle Assessment. Environmental Science \& Technology, 52(17), 9562-9578. https://doi.org/10.1021/acs.est.8b00967

Núñez, M., Civit, B. M., Muñoz, P., Arena, A. P., Rieradevall, J., \& Antón, A. (2010). Assessing potential desertification environmental impact in life cycle assessment. The International Journal of Life Cycle Assessment, 15(1), 67-78. https://doi.org/10.1007/s11367-009-0126-0

Payen, S., Civit, B. M., Golden, H., Niblick, B., Ubizeye, A., Winter, L., \& Henderson, A. D., (2019). Acidification \& Eutrophication. In R. Frischknecht \& O. Jolliet (Eds.), Global Guidance on Environmental Life Cycle Impact Assessment Indicators (Volume 2, pp. 60-79). UNEP; SETAC; Life Cycle Initiative. https://www.lifecycleinitiative.org/training-resources/global-guidancefor-life-cycle-impact-assessment-indicators-volume-2/

Perez, G. A. (2016). Antecedentes. In P. Garolera De Nucci, F. D. Mele, A. L. Nishihara Hun \& G. A. Perez (Eds.), Avances y estado de situación en análisis de ciclo de vida y huellas ambientales en la Argentina: Actas del V Encuentro Argentino de Ciclo de Vida y IV Encuentro de la Red Argentina de Huella Hídrica ENARCIV 2016 (1era ed., pp. 6-7). Ediciones INTA. ISBN: 978-987-521-810-9.

Pla Sentís, I. (1990). La degradación y el desarrollo agrícola de Venezuela. Agronomia Tropical (Venezuela). Serie Edafológica, 40(1-3), 7-27.

Potting, J., \& Hauschild, M. Z. (2006). Spatial differentiation in life cycle impact assessment: a decade of method development to increase the environmental realism of LCIA. The International Journal of Life Cycle Assessment, 11(1), 11-13. https://doi.org/10.1065/lca2006.04.005

Potting, J., Klöpffer, W., Seppälä, J., Risbey, J., Meilinguer, S., Norris, G., Lindfords, G. L., \& Goedkoop, M. (2001). Best available practice in life cycle assessment of climate change, stratospheric ozone depletion, photo-oxidant formation, acidification and eutrophication. Backgrounds and general issues.

Quirós, R. (2000). La eutrofización de las aguas continentales de Argentina. In A. Fernández (Ed.), El Agua en Iberoamérica: acuíferos, lagos y embalses (pp. 43-47). CYTED. Subprograma XVII. Aprovechamientos y gestión de recursos hídricos.

Rockström, J., Steffen, W., Noone, K., Persson, A., Chapin, F. S., Lambin, E. F., Lenton, T. M., Scheffer, M., \& Folke, C A. (2009). Safe Operating Space for Humanity. Nature, 461, 472-475. https://doi.org/10.1038/461472a

Schmid, A. G. (2008). Diferenciación espacial en la metodología de Análisis del Ciclo de Vida: desarrollo de factores regionales para eutrofización acuática y terrestre. Universidad de Santiago de Compostela. ISBN: 13: 978-84-612-8888-5.

Toffoletto, L., Bulle, C., Godin, J., Reid, C., \& Deschênes, L. (2007). LUCAS-A new LCIA method used for a Canadian-specific context. The International Journal of Life Cycle Assessment, 12(2), 93-102. https://doi.org/10.1065/lca2005.12.242

Udo de Haes, H. A., Finnveden, G., Goedkoop, M., Hertwich, E., Hofstetter, P., Klöpffer, W., Krewitt, W., \& Lindeijer, E. (2002). Life cycle impact assessment: striving towards best practice. SETAC Press Proceedings. 